\title{
Cellular immunity induced by a recombinant adenovirus- human dendritic cell vaccine for melanoma
}

Hadas Prag Naveh ${ }^{1}$, Lazar Vujanovic ${ }^{1}$ and Lisa H Butterfield ${ }^{1,2,3,4^{*}}$

\begin{abstract}
Background: Human Adenoviral vectors (HAdV) are immunogenic vectors which have been tested in many vaccination and gene therapy settings. Dendritic cells (DC) transduced by genetically engineered HAdV-5 (HAdV-5/DC), are investigational cancer vaccines being tested clinically. We have previously examined immune responses to HAdV-5 -encoded melanoma tumor antigens. Here, we determined whether the HAdV-5/DC also present immunogenic HAdV-5 vector-derived antigens, and characterized the cellular immune response to the viral as well as encoded melanoma tumor antigens.
\end{abstract}

Methods: Both $\mathrm{CD}^{+}$and $\mathrm{CD} 8^{+} \mathrm{HAdV}-5$-specific T cell responses were examined in vitro, with cells from both 8 healthy donors (HD) and 2 melanoma patients. PBMC were stimulated weekly with HAdV-5/DC and responses were examined after each stimulation. We also tested HAdV-5 neutralizing antibody levels and natural killer (NK) cell and regulatory T cell (Treg) activation and expansion in vitro.

Results: HAdV-5/DC rapidly induced a high frequency of type 1 cytokine producing HAdV-5-specific CD $8^{+}$and $\mathrm{CD}^{+} \mathrm{T}$ cells. IFNy and TNFa-producing T cells predominate. Those with pre-existing cellular memory to HAdV-5 had more robust responses to the HAdV-5 as well as tumor-associated antigens. NK cells are activated while Treg are only minimally and transiently expanded.

Conclusions: This study demonstrates that HAdV-5/DC promote strong type I cellular immunity to viral vector-derived antigens as well as to the encoded tumor antigens. The cytokine and chemokine milieu produced by HAdV-5/DC and the activated HAdV-5-specific T cells may enhance responses to encoded tumor antigens as well. These properties make HAdV-5/DC a cancer vaccine capable of activating type 1 virus and tumor antigen-specific immunity in a cooperative way.

Keywords: Adenovirus, Dendritic cells, T cells, Cytokines, Cancer vaccines

\section{Background}

Recombinant Human adenovirus serotype 5 (HAdV-5) is the most commonly used gene transfer vehicle with a long safety record [1]. The HAdV-5 $36 \mathrm{~kb}$ genome (double stranded DNA) is well characterized, and used in many experimental and clinical settings [2]. Its systemic use in vaccination and gene therapy has been limited by pre-existing immunity and the presence of neutralizing

\footnotetext{
* Correspondence: butterfieldl@upmc.edu

'Department of Medicine, University of Pittsburgh Cancer Institute, 5117

Centre Avenue, Suite 1.27, Pittsburgh, PA 15213, USA

${ }^{2}$ Department of Surgery, University of Pittsburgh Cancer Institute, 5117

Centre Avenue, Suite 1.27, Pittsburgh, PA 15213, USA

Full list of author information is available at the end of the article
}

antibodies in environmentally exposed individuals. Neutralizing antibodies, directed mainly against capsid hexon loops, are serotype specific. Approximately 45\% of adults in the United States possess neutralizing antibodies to HAdV-5. A variety of other Adenoviral subtypes and deletion mutants have been investigated (Ad28, Ad35 and "gutless" Adenovirus), in an attempt to circumvent pre-existing immunity in exposed individuals.

We have utilized E1/E3-deleted, replication deficient tumor antigen-encoding HAdV-5 in vitro and in vivo, to promote tumor antigen-specific immunity (reviewed in [3]. As previously shown, dendritic cells (DC) transduced with an HAdV-5 (HAdV-5/DC) encoding full length 
MART-1 (HAdV-5 MART1), activate MART-1 specific $\mathrm{CD}^{+}$and $\mathrm{CD} 4^{+} \mathrm{T}$ cells in vitro $[4,5]$ and in vivo [6]. Similarly, DC transduced with an HAdV-5 encoding alpha fetoprotein (AFP, HAdV-5hAFP) activate $\mathrm{CD} 8^{+}$ and $\mathrm{CD}_{4}^{+} \mathrm{T}$ cells to AFP in vitro, and are more efficient at $\mathrm{T}$ cell stimulation than AFP protein-fed DC [7-9]. Immunogenicity of $\mathrm{HAdV}-5 / \mathrm{DC}$ can be attributed to the fact that HAdV-5 transduction promotes a more mature DC surface phenotype, associated with a unique cytokine and chemokine secretion profile $[10,11]$. Additionally, transduction with HAdV-5 modulates components of MHC class I antigen processing machinery in DC [11]. Recently we showed that HAdV-5/DC can also promote NK cell chemotaxis in vitro and in vivo via IL-8/CXCL8 and IP-10/CXCL10 secretion and induce activation of both major subsets of NK cells, which requires cell-cell contact via cell surface TNF and IL-15 [12,13].

We have examined the cross-talk between HAdV-5 and DC, as well as downstream effects on transgenespecific $\mathrm{T}$ cell subsets and other lymphocytes. In addition to introducing a transgene, HAdV-5 engineering of DC also leads to expression of the remaining HAdV-5 genes encoded within the vector (derived from 28 genes and overlapping open reading frames) in the DC, and processing and presentation of viral coat proteins, such as the HAdV-5 hexon and fiber, released during HAdV-5 uptake in endosomes. The immunologic impact of the presentation of these viral proteins by transduced $\mathrm{DC}$ to $\mathrm{T}$ cells is yet unknown. Our previous preclinical murine models indicated that pre-immunization with HAdV-5 did not impact the antitumor immunity from an AdVMART-1-transduced DC vaccine [14], but detailed viral immunity assessments were not performed because mice are not permissive for HAdV infections.

Most previous studies examining humoral and cellular immunity to HAdV-5 have focused on directly injected vectors, and vectors involving HIV antigens encoded by HAdV-5. Humoral responses to HAdV-5 were shown to be critical to the efficacy of an HIV vaccine [15]. It has been shown that HAdV-5 neutralizing antibody levels were unrelated to $\mathrm{T}$ cell responses to hexon or E2A viral proteins, and that the levels of HAdV-5specific $\mathrm{CD} 4^{+} \mathrm{T}$ cell responses varied with the specific deletions in the HAdV-5 backbone [16]. Multiplex cytokine profiling showed that a broad Th1/Th2/regulatory profile resulted from MRKAd5 HIV gag immunization of healthy volunteers [17].

Other previous clinical studies utilizing recombinant HAdV-5 vectors encoding additional foreign viral antigens (HIV, EBV, CMV) have examined some aspects of immune response to the HAdV-5 viral antigens $[14,15,18-23]$. Other reports in the literature utilize replication-competent HAdV-5 which still encode the
E1a/E1b transactivators (including oncolytic viruses), leading to a high level of viral gene transcription and translation, and often lytic growth in infected human cells. However, the responses to recombinant HAdV-5 which encode normal, non-mutated self-antigens, like melanoma lineage tumor antigens, may have a unique profile due to the colocalized presentation of both classes of antigens (self and viral). Such self-antigen encoding HAdV - 5 have been tested in transduced DC clinical trials $[6,22,23]$, but the immunity to the HAdV- 5 aspects of these vaccines has not been examined to date.

We have recently developed a new melanoma vaccine clinical trial testing immunization with three full length melanoma tumor antigens (HAdV-5 TMM2, encoding Tyrosinase, MART-1 and MAGE-A6), instead of a single antigen [24], as in our previous trial [6]. While we have previously characterized the $\mathrm{T}$ cell responses to the encoded tumor antigens, we have not examined whether HAdV-5-specific cellular immune responses are also activated. Based on previous studies showing that the HAdV-5 capsid protein hexon encodes CD8 and CD4 T-cell epitopes $[25,26]$, we hypothesized that HAdV-5specific memory $\mathrm{T}$ cells may be detected in the periphery of environmentally exposed individuals, and that these responses might be quickly reactivated with HAdV-5/DC stimulation. We also hypothesized that these responses would be predominantly type 1 , which might serve to skew the cellular environment in which the virally encoded tumor antigens are presented. Here, we have carefully examined the in vitro $\mathrm{CD}^{+}$and $\mathrm{CD} 4^{+} \mathrm{T}$ cell response to HAdV-5-specific antigens on DC transduced with a replication-deficient HAdV-5 (HAdV-5 TMM2). We find that a high frequency of type $1 \mathrm{CD}^{+}$and $\mathrm{CD} 4^{+}$ $\mathrm{T}$ cells are activated to the viral antigens and the overall cytokine milieu is type 1 . We also find that NK cells in culture are activated, and regulatory $\mathrm{T}$ cells (Treg) remain at a low frequency in vitro, while activated $\mathrm{CD} 4^{+} \mathrm{T}$ cells expand. Importantly, HD and melanoma patients with pre-existing cellular HAdV-5-specific memory $\mathrm{T}$ cells showed potent $\mathrm{HAdV}-5$ and melanoma antigen responses in vitro which were unrelated to humoral memory.

\section{Results and discussion}

\section{HAdV-5/DC rapidly induce high frequencies of} HAdV-5-specific $\mathrm{CD}^{+}{ }^{+} \mathrm{T}$ cells

To define the $\mathrm{CD} 4^{+} \mathrm{T}$ cell response to HAdV-5 in HD, we stimulated PBMC with HAdV-5 TMM2-transduced DC (HAdV-5 TMM2/DC) in vitro, for a total of three weekly stimulations. This was chosen to mimic the three total vaccinations used in our current and previous clinical trials $[6,27,28]$. We hypothesized that we would expand high frequencies of type $1 \mathrm{~T}$ cells, based in part on the HAdV-5 virus-specific $T$ cell adoptive transfer studies [20] which have demonstrated that HAdV-5-specific 
$\mathrm{CD}^{+}$and $\mathrm{CD} 4^{+} \mathrm{T}$ cells can have a high proliferative capacity, secrete IFN $\gamma$ and show cytotoxicity towards HAdV-5-infected targets [21]. T cells were tested weekly, after each stimulation. We utilized two standardized, functional assays to measure responses, using the cytokine ELISPOT from purified T cell subsets, and flow cytometry with surface and intracellular cytokine staining. Mean HAdV-5-specific CD4 $4^{+} \mathrm{T}$ cell frequencies for $7 \mathrm{HD}$ are shown in Figure $1 \mathrm{~A}, 1 \mathrm{~B}$, and $1 \mathrm{C}$.

While several promiscuous MHC class II epitopes have been described ( $\mathrm{Hex}_{571-600}, \mathrm{Hex}_{856-885}, \mathrm{Hex}_{901-930}$ ) [29], we primarily detected responses to the HAdV-5 hexon ${ }_{901-930}$ (Hex $901-930$ ) epitope. As shown in Figure 1A, after only a single HAdV-5 TMM2/DC stimulation, a high frequency of $\mathrm{Hex}_{901-930^{-}}$-specific $\mathrm{CD}^{+}{ }^{+} \mathrm{T}$ cells were expanded, producing primarily IFN $\gamma$, as well as TNF $\alpha$ and IL-2. Low frequencies of IL-10 producing cells were also detected from HD. TNF $\alpha$ or IL-10 responses to the $\mathrm{Hex}_{571}$ and $\mathrm{Hex}_{856}$ epitopes were not detected and there were few examples of IFN $\gamma$ or IL-2-producing $\mathrm{T}$ cells detected specific to $\mathrm{Hex}_{856}$ (not shown). Since the HAdV-5 hexon capsid protein is very immunogenic and has been found to account for the majority of anti-HAdV-5 $\mathrm{T}$ cell reactivity in other studies [21], we hypothesized that other HAdV-5-derived epitopes might play an important role in $\mathrm{HAdV}-5 / \mathrm{DC}$ induced $\mathrm{T}$ cell responses. We used an E1/E3-deleted HAdV-5 encoding $\beta$-galactosidase (HAdV-5 LacZ), which has the same HAdV-5 backbone as HAdV-5 TMM2 and which also encodes the same 28 overlapping HAdV-5 gene products as HAdV-5 TMM2, to measure the total HAdV-5-specific $\mathrm{T}$ cell responses. While the hexon-specific responses peaked at one week (all measured cytokines), the total HAdV-5 response, largely consisting of IFN $\gamma$ and TNF $\alpha$-producing $\mathrm{T}$ cells, continued to increase through three weekly stimulations (Figure 1B). The difference in expansion kinetics between hexon-specific and total HAdV-5specific $\mathrm{CD}_{4}^{+} \mathrm{T}$ cells may be due to the minimal levels of viral gene transcription and the presence of intact hexon protein in the coat of AdVTMM2. Comparing

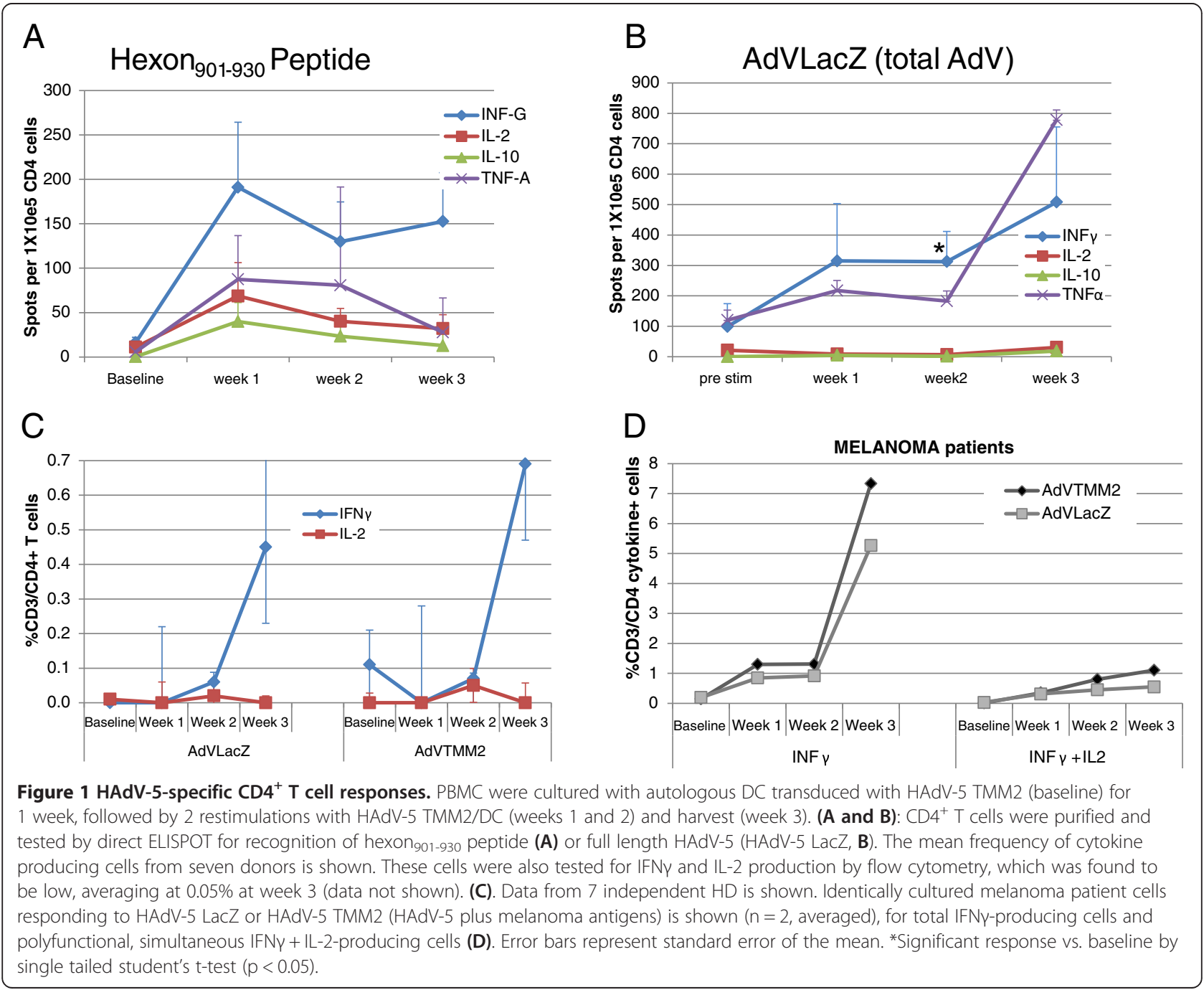


the $\mathrm{CD} 4^{+} \mathrm{T}$ cell response to the HAdV-5 backbone (HAdV-5 LacZ) with the response to both HAdV-5 and the three melanoma-associated antigens (MAA, HAdV-5 TMM2), a minimal IL-2 response was detected at week 2 and a stronger IFN $\gamma$ response was observed after three stimulations, with specificity for both viral and melanoma antigens (Figure 1C). The frequency of $\mathrm{CD}^{+} \mathrm{T}$ cells which were polyfunctional (producing IFNY and IL-2 simultaneously by flow cytometry) was low, averaging $0.05 \%$ of $\mathrm{CD}^{+}{ }^{+} \mathrm{T}$ cells at the peak at week 3 (not shown).

Cancer patients can be immune suppressed, due to multiple immune modulatory effects of both tumor cells and many standard of care cancer treatments. Therefore, it was important to include cancer patient cells in the analysis. We used IFN $\gamma$-producing HAdV-5-specific $\mathrm{CD} 4^{+} \mathrm{T}$ cells obtained from two different melanoma patients also expanded to high frequencies in vitro. Despite these virus-specific $T$ cell frequencies, the $T$ cells specific to the three encoded melanoma antigens were still detectable, at over 1\% frequency, at week 3 (Figure 1D). Interestingly, the frequency of polyfunctional $\mathrm{CD} 4^{+} \mathrm{T}$ cells was higher in both of the patient cell cultures $(0.3$ and $0.8 \%$ at week 3 , not shown) than in HD cultures, indicating that the tumor and therapeutic treatments received did not inhibit HAdV-5 or tumor antigen responses. Conceivably, immunotherapies received by these patients may have supported previous expansion of the melanoma antigen-specific $\mathrm{T}$ cells, allowing for memory responses.

\section{HAdV-5-specific $\mathrm{CD}^{+} \mathrm{T}$ cell responses are rapidly induced by HAdV-5/DC}

To define the $\mathrm{CD}^{+} \mathrm{T}$ cells response to HAdV-5 in HD, we utilized the same culture and assay strategy. We tested the HLA-A2-restricted hexon ${ }_{711-721}$ peptide previously identified as the immunodominant hexon epitope, but did not reliably detect responses from any of our HLA-A2 ${ }^{+}$ donors (data not shown). We did detect high frequency type 1 responses to the total HAdV-5 proteins (HAdV-5 LacZ) as shown in Figure 2. Cells producing TNFa and IFN $\gamma$ predominated and showed greater frequencies with every subsequent stimulation, with IL-2 producing cells also detected (Figure 2A). Comparing the HAdV-5 and HAdV- $5+$ MAA responses, $\mathrm{CD} 8^{+} \mathrm{T}$ cells producing IFN $\gamma$ and IL-2 (at a lower frequency) could be detected to both after 3 stimulations (Figure 2B). Comparing $\mathrm{CD}_{4}^{+}$and $\mathrm{CD}^{+} \mathrm{T}$ cell responses, there were more IFN $\gamma$-producing $\mathrm{CD} 4^{+} \mathrm{T}$ cells and more IL-2-producing $\mathrm{CD}^{+} \mathrm{T}$ cells expanded from the HD tested (Figures 1C and $2 \mathrm{~B}$ ). The frequency of polyfunctional HAdV-5-specific $\mathrm{CD}^{+}{ }^{+}$cells was also low, averaging $0.04 \%$ of $\mathrm{CD}^{+} \mathrm{T}$ cells at the peak at week two (not shown).

To confirm that the patterns observed in HD were representative of the responses in melanoma patients, we repeated the experiments with PBMC from the same HAdV-5anced stage melanoma patients (Figure 2C). While HAdV-5-specific cells were clearly expanded, the relative frequency of melanoma antigen to HAdV-5 viral antigen responses was much greater than in $\mathrm{CD} 4^{+} \mathrm{T}$ cells (Figure 1C). The frequencies of polyfunctional $\mathrm{CD}^{+} \mathrm{T}$ cells in the two patients were similar to that of the HD $(0.05 \%$, peak at week 1 , not shown).

\section{Impact of HAdV-5-specific cellular memory responses}

Because most individuals have been previously exposed to $\mathrm{HAdV}-5$ in the environment, we expected that donors and patients would have variable but detectable levels of pre-existing cellular and humoral HAdV-5 immunity. Additional file 1: Figure S1 shows the range of antiHAdV-5 neutralizing antibodies detected in our pool of donors and patients. These data show that the majority of donors and patients have been exposed to HAdV-5 and have detectable titers of neutralizing antibodies, as expected. The two melanoma patient's sera did not differ appreciably from the HD sera tested. Importantly, the baseline titer of anti-HAdV-5 neutralizing antibodies did not correlate with the induced cellular response to HAdV-5 in vitro. This is similar to the observation of Koup et al. [16] in their study of intramuscular HAdV5-HIV gag immunization.

We next determined the impact of baseline cellular immunity to HAdV-5 on the resultant expansion of T cells from HAdV-5/DC stimulation. By intracellular cytokine staining, all tested donors had at least $0.02 \%$ positive IFN $\gamma$ or IL-2 producing $\mathrm{CD}^{+}$and/or $\mathrm{CD}^{+}{ }^{+} \mathrm{T}$ cells, and the assay sensitivity is reported to be $0.01 \%$ or less [30]. To investigate the impact of baseline HAdV-5-specific cellular memory on the level of induced $\mathrm{T}$ cell responses to HAdV-5 antigens as well as MAA, we used a cut off of $0.03 \%$ baseline $\mathrm{CD} 8^{+}$or $\mathrm{CD} 4^{+}$positivity (IFNY or IL-2) to compare the induced $\mathrm{T}$ cell responses between those with $\leq 0.02 \%$ positive HAdV-5-specific T cells (the lowest detected), to those with $\geq 0.03 \%$ positive HAdV-5-specific $\mathrm{T}$ cells. As shown in Figure 3, those with cellular memory responses (red symbols) generally induced higher frequency $\mathrm{CD}^{+}$IFN $\gamma$ and IL-2 responses to HAdV-5 antigens and MAA after the three stimulations (Figure 3A-B). This baseline memory group also showed higher $\mathrm{CD} 4^{+}$ IFNY responses at 2-3 weeks to the HAdV-5 and HAdV-5 TMM2 (HAdV-5 + MAA) groups. The melanoma antigen-specific responses were stronger (with both IFN $\gamma$ and IL-2) from those with baseline HAdV-5 cellular memory (Figure 3A-D). Importantly, the IL-2 producing $\mathrm{T}$ cells recognizing autologous DC pulsed with lysate from the melanoma cell line Mel526 (expressing Tyrosinase, MART1, MAGE-A3/6 antigens, not shown) were also most frequently recognized by those with HAdV-5 cellular memory (Figure 3E). These data suggest that the higher 


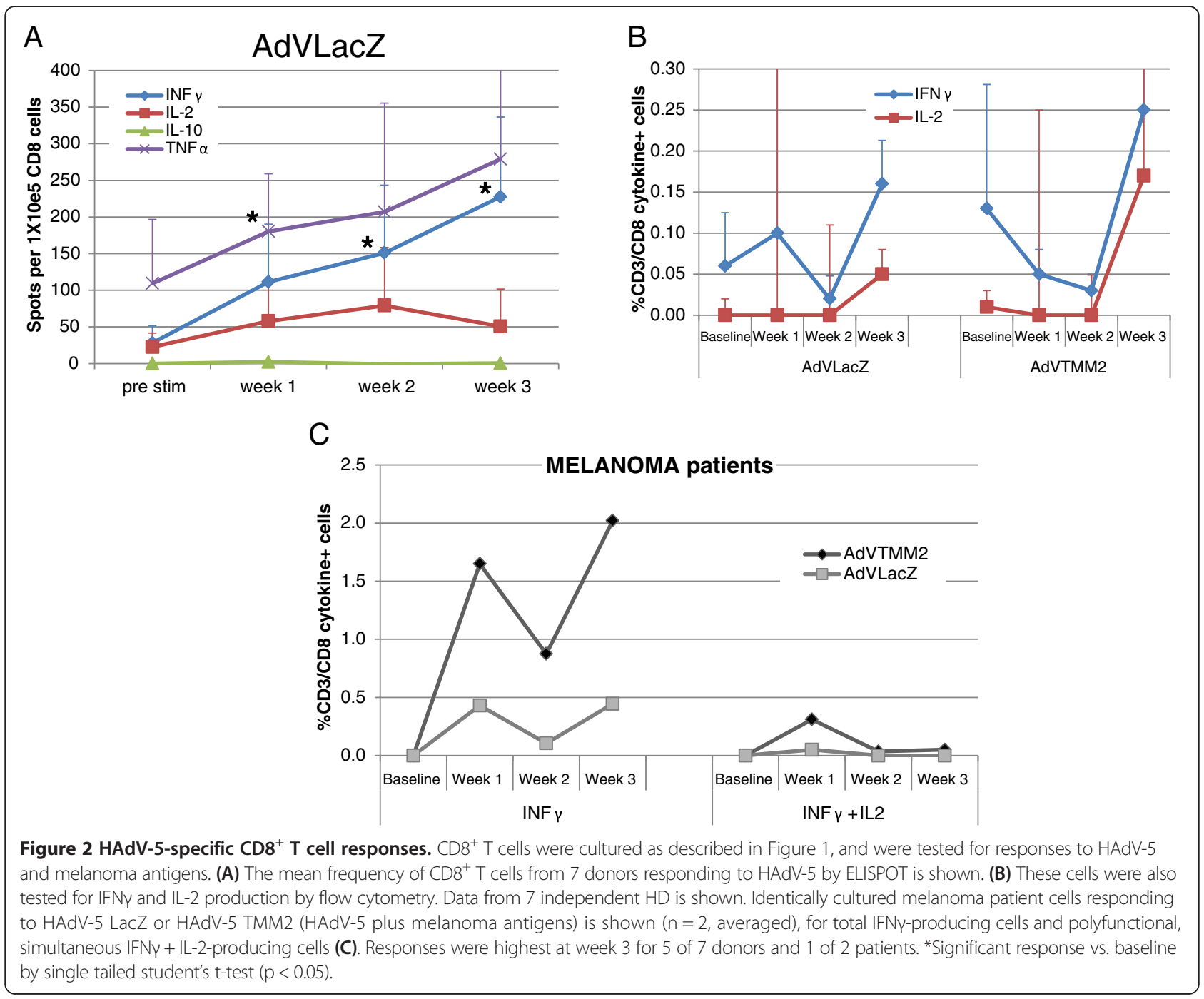

frequency of HAdV-5-specific cells producing type 1 cytokines provides a supportive milieu for the MAAspecific $\mathrm{T}$ cells also being stimulated by the HAdV-5 TMM2/DC.

Our observation of robust expansion of type 1 cytokine producing HAdV-5-specific $\mathrm{T}$ cells is in sharp contrast to the lack of expansion of HAdV-5-specific T cells in vivo after HAdV-5-HIV gag vaccination [16]. This may relate in part to the difference in HAdV-5 backbone. The HIV gag-encoding vector in that study was not only E1 and E3 deleted, but also E4 deleted.

\section{HAdV-5 TMM2/DC promote NK cell activation in vitro}

Our previous data showed that HAdV-5 LacZ/DC can activate NK cells [12]. Therefore, we examined the activation status of NK cell subsets in the HAdV-5 TMM2/ DC cultures. The NK cells were separately analyzed as two major and three minor subsets (Additional file 2: Figure S2a), as described previously [31]. We observed that the frequency of total activated $\mathrm{CD} 69^{+} \mathrm{NK}$ cells (Additional file 2: Figure S2b) and activated IFN $\gamma$ producing NK cells (Additional file 2: Figure S2c) increased with HAdV-5 TMM2/DC stimulation. These data confirm that HAdV-5 TMM2/DC promote NK cell activation, and that changing the encoded transgene from xenoantigens $\beta$-galactosidase or luciferase to melanoma tumor antigens tyrosinase, MART-1 and MAGE-A6 does not change the ability to activate NK cells.

\section{HAdV-5 TMM2/DC do not induce regulatory $T$ cell responses}

There have been reports of certain types of DC activating suppressive Treg responses [32]. Such expansion of Treg would be an unwanted side effect of HAdV-5/DC stimulation in a cancer immunotherapy setting. Therefore, we determined the overall levels of both $\mathrm{CD} 4^{+} \mathrm{T}$ cell activation (regardless of antigen specificity), as well as levels of Treg in the HAdV-5/DC cultures by flow 

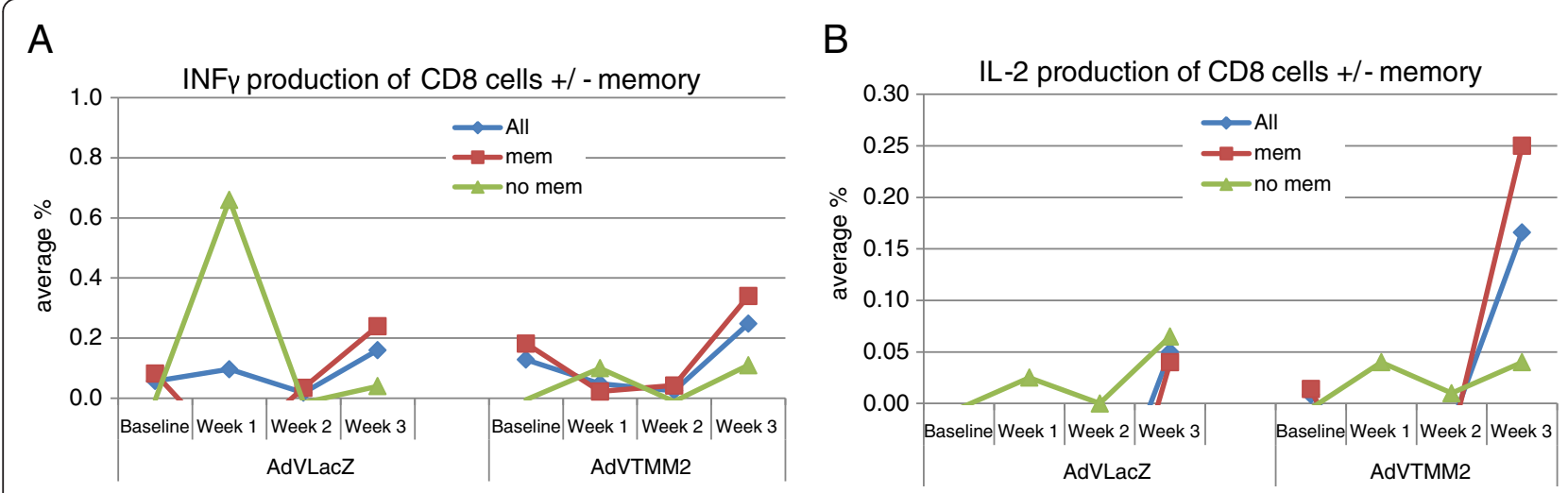

C

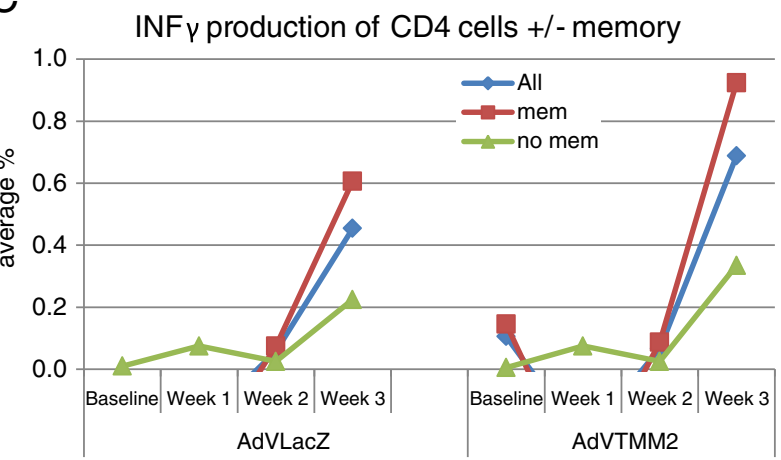

$\mathrm{D}$

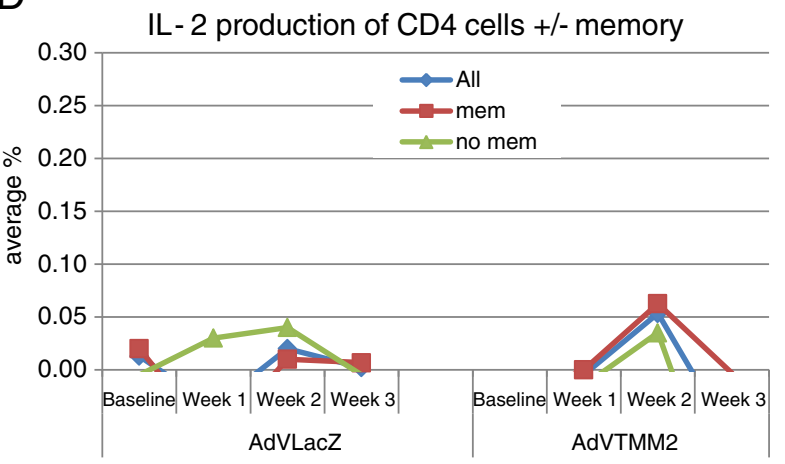

$E$

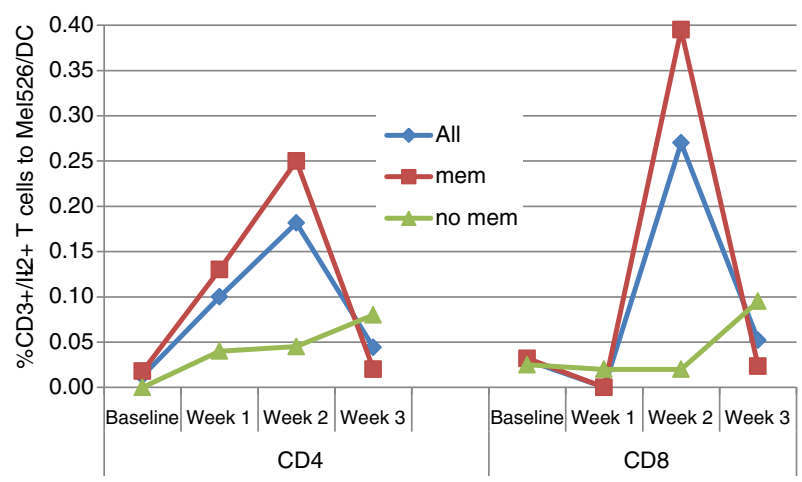

Figure 3 Impact of baseline memory on induced responses. The frequency of $C D 8^{+}$( $A$ and $B$ ) and $C D 4^{+}(C$ and $D) T$ cells producing IFNY (A and C) or IL-2 (B and D) to HAdV-5 (HAdV-5 LacZ) or HAdV-5 + melanoma antigens (HAdV-5 TMM2) are shown ("All"). Those HD with $\geq 0.03 \%{ }^{+}$ baseline IFNy and/or IL-2 responses were also analyzed separately, as having cellular memory responses ("mem", $n=5$ ) vs. those with $<0.03 \%{ }^{+}$cells, or no baseline cellular memory ("no mem", $n=2$ ). The frequency of $C D 8^{+}$or $C D 4^{+} T$ cells producing IL-2 to DC loaded with Mel526 lysate are shown (E).

cytometry. As shown in Figure 4A and B, the frequency of activated $\left(\mathrm{CD} 25^{+}\right) \mathrm{CD} 4^{+} \mathrm{T}$ cells were sharply increased after a single stimulation with HAdV-5/DC to $15-40 \%$ of the total $\mathrm{CD}_{4}^{+} \mathrm{T}$ cells, later decreasing to $8-27 \%$. In contrast, the frequency of $\mathrm{CD} 25^{\text {hi }}$ FoxP3 $^{+} \mathrm{CD}^{+}$Treg detected was extremely low $(<4 \%)$, and decreased to $<1 \%$ after the third HAdV-5/DC stimulation (Figure 4C). This could be attributed to the fact that HAdV-5/DC have a unique level of maturation where they secrete low amounts of immunoregulatory cytokines (IL-10 and IL-
13), and more immunostimulatory cytokines (TNF $\alpha$, IL-15) [11].

\section{Cytokine and chemokine milieu}

As shown in Figures 1 and 2, we detected high frequencies of HAdV-5-specific $\mathrm{T}$ cells producing primarily IFNy and TNF $\alpha$. We also measured CD69 on all T cells, to determine the activation status on a population basis. At baseline, $2 \%$ of $\mathrm{CD}_{4}^{+} \mathrm{T}$ cells and $4 \%$ of $\mathrm{CD} 8^{+} \mathrm{T}$ cells were $\mathrm{CD}_{69} 9^{+}$, and $\mathrm{PMA} /$ ionomycin activation resulted in 


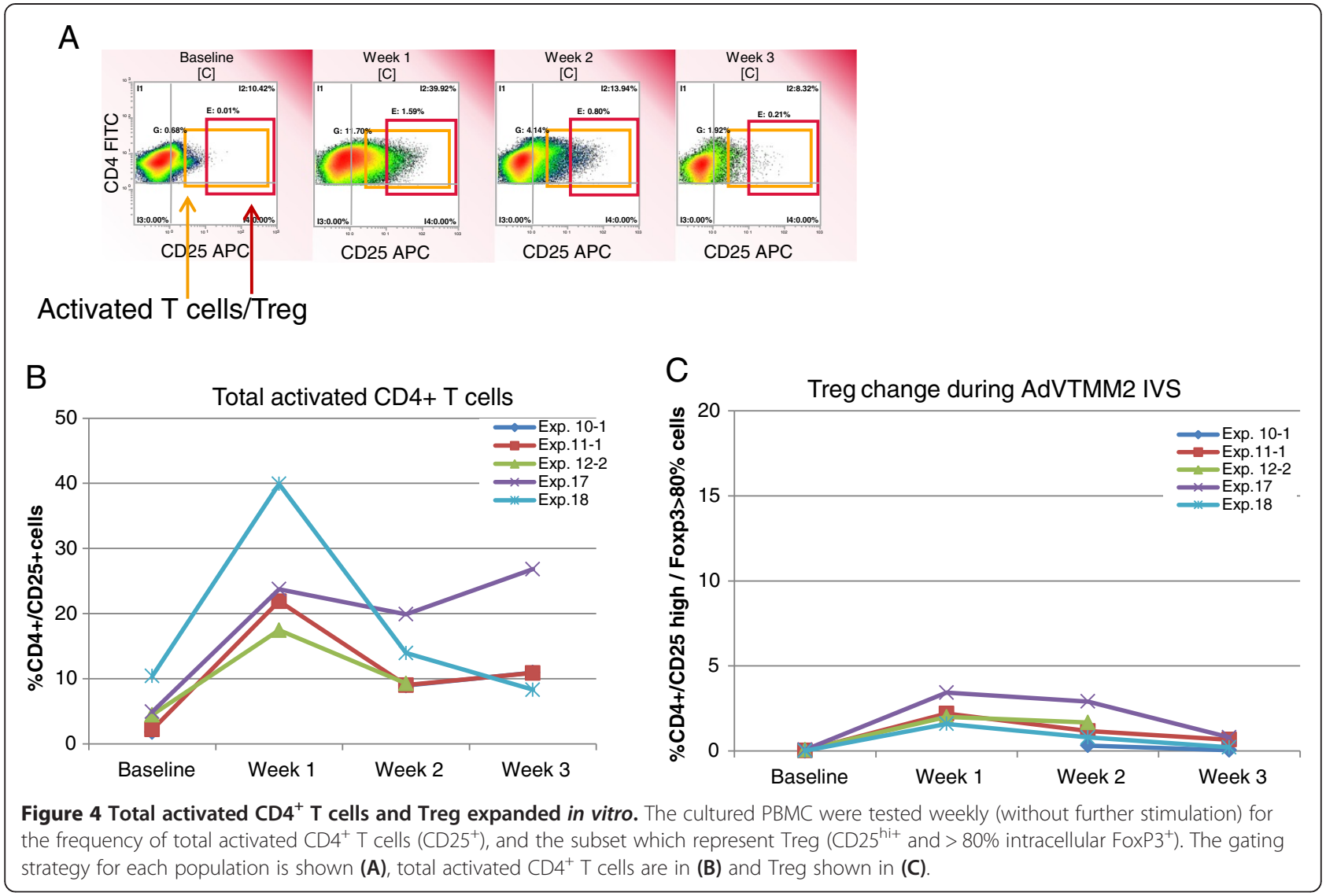

45-55\% CD69 positivity (data not shown). The majority (up to $89 \%$ ) of IFNy and/or IL-2-producing cells were $\mathrm{CD} 9^{+}$, as expected. Of the $11-24 \%$ which expressed CD69 following HAdV-5 LacZ/DC exposure, less than $1 \%$ generally produced IFN $\gamma$ or IL-2 (Figures 1 and 2). Therefore, we hypothesized that additional cytokines were likely being produced by these $\mathrm{CD}^{+} 9^{+}$activated $\mathrm{T}$ cells. To more broadly characterize the cytokine milieu (created by both HAdV-5/DC and lymphocytes), we tested many soluble factors present in the HAdV-5/DC cultures at each weekly stimulation by multiplex Luminex assay. As shown in Figure 5, many cytokines and chemokines were detected. In addition to IFN $\gamma$ and TNF $\alpha$, MCP-1/CCL2, RANTES/CCL5, IL-7 and GM-CSF were highly produced. IL-7 has anti-apoptotic properties and may also serve to support T cell growth and GM-CSF may aid DC viability [33,34]. Based on our recent findings on NK cell crosstalk, we examined both CXCL8/IL-8 and CXCL10/IP-10, which we demonstrated are secreted by $\mathrm{HAdV}-5 / \mathrm{DC}$ and responsible for NK cell chemotaxis [13]. Both were detected at high levels, which would support NK cell chemotaxis at each weekly stimulation (as well as possibly chemotaxis of neutrophils and type 1 T cells, respectively). IL-15, which we showed is crucial for HAdV-5/DC-mediated NK cell activation (when trans- presented on the DC surface), and which has also been shown to support memory $\mathrm{T}$ cell homeostasis was also detected [35].

There have been reports of type 1 interferons (IFN) produced by a variety of human cells upon HAdV-5 exposure, which might be expected as part of an antiviral response [36]. HAdV-5 induction of IFN $\alpha$ has been shown to have a variety of positive and negative effects in the setting of recombinant $\mathrm{HAdV}-5$ vaccines [37]. IFN $\alpha$ is also known to help mature $\mathrm{DC}$, promote $\mathrm{CD}^{+} \mathrm{T}$ cell growth, support memory responses, and support NK cells [38]. In these in vitro cultures, we detected low levels of IFN $\alpha$, generally under $100 \mathrm{pg} / \mathrm{mL}$. These levels (which are well below physiologically achieved levels in high dose IFN $\alpha$-treated patients) may be too low to promote DC maturation [10].

We did not detect VEGF or IL-2 by Luminex. As shown in Figures 1 and 2, IL-2-producing T cells were detected by intracellular cytokine staining, but levels of soluble IL-2, a potent $\mathrm{T}$ cell growth factor, were either below detection or more likely taken up by other cells in culture. In contrast, soluble IFN $\gamma$ and $\mathrm{TNF} \alpha$, also produced by antigen-specific $\mathrm{T}$ cells, were within detection limits in the cultures. VEGF is a potent inducer of angiogenesis and skews immunity to support tumor growth, hence the lack 


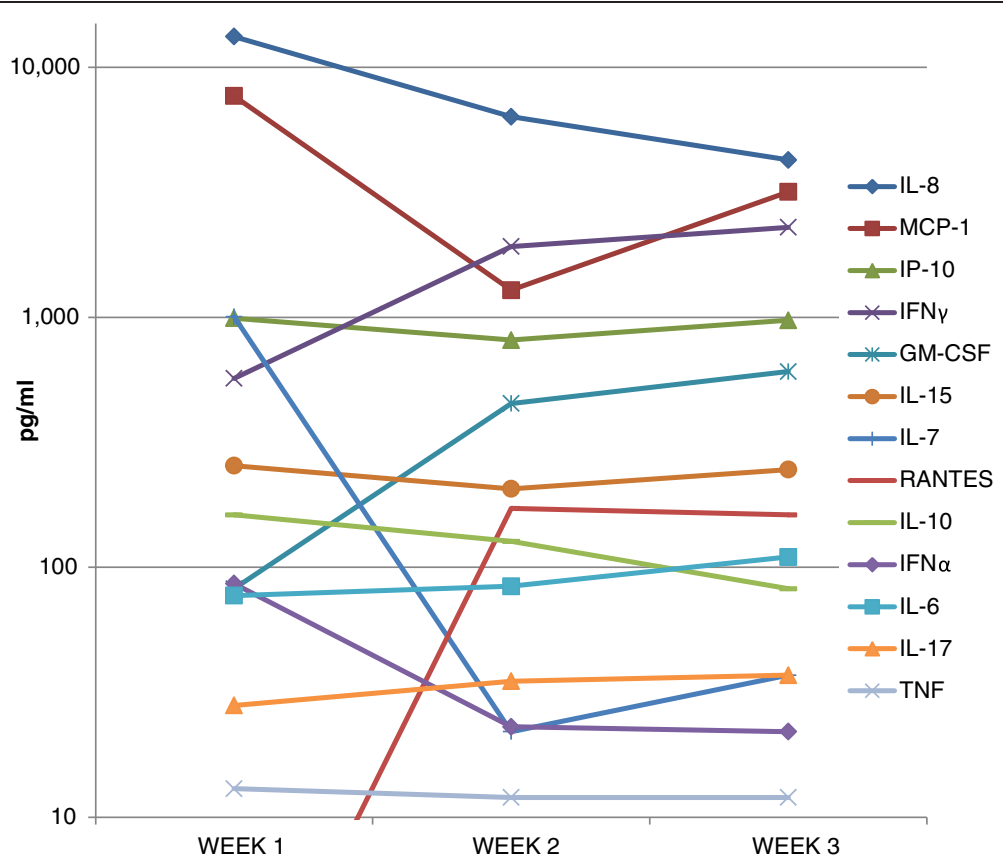

Figure 5 Analytes in weekly HAdV-5 TMM2/DC cultures by Luminex. Cell-free supernatents were collected after each 7 day culture period and frozen until simultaneous testing by multiplex Luminex 30-plex assay. The average levels for each analyte detected are shown at each time point, pooling 4-7 HD and 2 melanoma patients' culture data.

of VEGF is a positive aspect of the HAdV-5/DC cross talk with lymphocytes. Lastly, we detected low levels of IL-17, which may be produced by pro- or anti-tumor T cells [39]. The role of Th17 cells in our system is not yet known, but these preclinical data suggest that these cells should be tested in our clinical trial patients.

When comparing HD to melanoma patient (results not shown), the patients had lower levels of IL-10, IL-6, IL-15, MCP-1, IL-Ira, IP-10, MIG and IL-8 and higher levels of MIP-1 $\alpha$ and GM-CSF, although higher numbers of HD and patients are needed to determine the significance of these observations. We did not detect a correlation between neutralizing antibody levels and cytokines produced in HD or patient cell cultures.

A study of $\mathrm{HD}$ vaccinated intramuscularly with MRKAd5-HIV-1 gag tested the cytokine profiles of PBMC at 30 weeks post injection [17]. The array of analytes tested was similar to ours. IL-8, MCP-1 and IP-10 were found in similar concentrations and were also the highest produced analytes in their study, supporting the potential in vivo relevance of our findings. However, there were also several differences between their findings and ours, including higher levels of IFN $\gamma$, GM-CSF, lower levels of IL-6 and IL-10 and no VEGF detected in our study. It will be important to confirm the quality and frequency of these cellular and secreted molecule responses presented with the in vivo responses in patients vaccinated with HAdV-5 TMM2/DC.

\section{Conclusions}

We find that DC engineered with a replication-deficient Ad5 vector encoding three melanoma antigens activate high frequencies of HAdV-5-specific $\mathrm{CD} 4^{+}$and $\mathrm{CD} 8^{+}$ $\mathrm{T}$ cells. These $\mathrm{T}$ cells produce both IFN $\gamma$ and TNF $\alpha$ effector cytokines, which create a type 1 cytokine milieu, and which have recently been shown to be important to promote senescence in tumors [40]. These cellular responses were independent of the level of anti-HAdV-5 humoral immunity. Interestingly, those with pre-existing cellular memory to HAdV-5 had superior responses not only to the HAdV-5 but also to the melanoma antigens. This suggests that the type 1 virus-specific $\mathrm{T}$ cell response may support a response to the vector-encoded melanoma antigens. We will investigate this in our ongoing HAdV-5 TMM2/DC vaccine clinical trial, in vivo. Additionally, we reaffirmed the ability of HAdV-5/DC to activate NK cells and not Treg, which can further aid in the creation of the type 1-skewing milieu, as well as provide an innate cytotoxic response against tumors.

\section{Methods}

\section{Cell culture and cell lines}

Mel526 is a human melanoma cell line which expresses MART-1/Melan-A, tyrosinase and MAGE-A3/6. The cell lines (Mel526, HEK293, A549) were cultured in Advanced RPMI 1640 medium supplemented with 10\% fetal bovine serum (FBS), 1\% penicillin streptomycin and 1\% L-glutamine (all reagents from Invitrogen), in a humidified 
$37^{\circ} \mathrm{C}$ incubator under $5 \% \mathrm{CO}_{2}$ tension. Mel526 lysate was prepared by repeated freeze/thaw of a cell pellet.

\section{Peptides}

Peptides were synthesized at the University of Pittsburgh Peptide Synthesis Facility using standard f-moc technology. Stock solutions were prepared in DMSO $(10 \mathrm{mg} / \mathrm{mL})$ and were kept at $-80^{\circ} \mathrm{C}$ until use. HAdV-5 hexon ${ }_{711-721}$ TFYLNHTFKK and the HAdV-5 promiscuous hexon peptides: $571-600$ NLLLLPGSYTYEWNFRKDVNMVLQSS LGND, 856-885 VDSITQKKFLCDRTLWRIPFSSNFMSM GAL and 901-930 LDMTFEVDPMDEPTLLYVLFEVFDV VRVHR.

\section{Virus amplification, purification and titer}

Virally infected HEK 293 cells (ATCC, \#CRL-1573) were freeze/thawed and the cleared supernatant was layered onto a CsCl step gradient $(1.2 \mathrm{~g} / \mathrm{mL} / 1.4 \mathrm{~g} / \mathrm{mL})$ and centrifuged at 35,000 rpm $1.5 \mathrm{~h}$. The virus band was dialyzed (Pierce Slide-a-lyzer $10 \mathrm{~K}$, Tris/MgCl2/glycerol), and concentrated (Millipore) in formulation buffer $(2.5 \%$ glycerol (w/v), $25 \mathrm{mM} \mathrm{NaCl}$ and $20 \mathrm{mM}$ Tris- $\mathrm{HCl}$, $\mathrm{pH}$ 8.0; Clontech), aliquoted and titered (Adeno-X Rapid Titer Kit, Clontech). HAdV-5 LacZ was obtained from the University of Pittsburgh Vector Core. HAdV-5 TMM2 encodes a CMV-Tyrosinase-IRES-MART-1-SV40pA cassette in the E1 region, and an RSV-MAGE-A6-SV40pA cassette in the E3 region [24].

\section{RT-PCR}

Total RNA was isolated (Qiagen Kit; Qiagen) and quantified by UV spectrophotometry. RT-PCR was performed using Random Primers, and SuperscriptTM III Reverse Transcriptase, Ampli Taq Gold polymerase. Sequencedspecific primers were as follows:

Tyrosinase, Forward: 5'-ATT-CCA-TAT-TGG-GACTGGCGG-GAT-3'/Tyrosinase

Reverse: 5'CAA-TGG-GTG-CAT-TGG-CTT-CTGGAT-3'

MART-1, Forward: 5' - TGC-AGA-TAT-CCA-TCACACTGG-3'

Reverse: 5' - GGA-GGG-GCA-AAC-AAC-AGA T - 3' MAGE-A6, Forward: 5'- AGT-AGG-AAG-GTG-GCCAAGTTG-GTT-3'

Reverse: 5' - TAT-TGG-GTG-AGC-TTC-TTGGGA-3' $\beta$-Actin, Forward: 5'-GGC-ATC-GTG-ATG-GACTCC-G-3'

Reverse: 5'-GCT-GGA-AGG-TGG-ACA-GCG-A-3'

All primers were prepared by Integrated DNA Technologies, Inc. PCR reactions for $\beta$-Actin, Tyrosinase and MART-1 were 32 cycles $\left(61.7^{\circ} \mathrm{C}\right.$ annealing), and for MAGE-A6, 30 cycles $\left(55^{\circ} \mathrm{C}\right.$ annealing) were used.
Isolation of peripheral blood mononuclear cells (PBMC) Peripheral blood was obtained from 8 different HLA$\mathrm{A} 2^{+/-} / \mathrm{HLA}_{-\mathrm{DR}}{ }^{+/-}$normal donors with their written consent, under an IRB-approved protocol (UPCI 04-001, L. H. Butterfield, PI).

Melanoma patient PBMC were obtained under Protocol UPCI 96-099 (J. M. Kirkwood, PI) at a time point distant from any treatment. Patient \#1 was diagnosed at stage 1, and 5 years post diagnosis, an inguinal metastases was found and she was treated with lymphadenectomy + high dose Interferon alpha-2b. Patient \#2 was diagnosed at stage 4 and received several previous treatments: Temozolomide, Interferon, high dose IL-2, BCG plus tumor vaccine. PBMC were separated from blood using FicollHypaque gradient centrifugation (Cellgro; Mediatech, Inc.) with average viability of $98 \%$. Additional buffy coats from healthy donors were obtained from the Central Blood Bank (Pittsburgh, PA).

\section{Generation of dendritic cells (DC) and DC culture}

DC were prepared as described [24] with some modifications. Mononuclear cells $\left(7-8 \times 10^{7}\right)$ were plated in T-75 flasks (Costar) in RPMI 1640/PSF/5-10\% human AB serum for $2 \mathrm{~h}$ at $37^{\circ} \mathrm{C}$ in a humidified $\mathrm{CO}_{2}$ incubator. The non-adherent cells were removed by gentle rinsing with PBS, and the loosely-adherent cells were cultured in medium with $800 \mathrm{U} / \mathrm{mL}$ GM-CSF (Sargramostim; Amgen) and $500 \mathrm{U} / \mathrm{mL}$ IL-4 (Schering Plough) for 6-7 d. The DC were harvested by TrypLE select, and had average $90 \%$ viability at harvest, and $85 \%$ viability post HAdV-5 transduction. DC were either transduced at moi $=500$ pfu: DC or loaded with Mel526 lysate at $10 \mu \mathrm{g} / \mathrm{ml}$ for 2 hours at $37^{\circ} \mathrm{C}$ in serum-free medium, then washed.

\section{In vitro stimulation}

PBMC from $\mathrm{HD}$ or patients were stimulated in vitro using HAdV-5/TMM2 transduced DC in a ratio of 1:10-1:40 (DC:PBMC). $25 \mathrm{ng} / \mathrm{ml}$ of IL-7 was added once at setup of the IVS. $30 \mathrm{U} / \mathrm{ml}$ IL-2 was added on day 3 of each IVS to maintain viability. Stimulated PBMC were collected weekly, washed, and re-stimulated with freshly transduced DC. A portion of PBMCs were collected from culture weekly and cryopreserved.

\section{Selection of $\mathrm{CD}^{+}$and $\mathrm{CD} 4^{+}$lymphocytes}

Before ELISPOT assays, the $\mathrm{CD}^{+}$and $\mathrm{CD}^{+}{ }^{+} \mathrm{T}$ cells were sequentially isolated by positive and negative MACS, respectively, with the use of immunomagnetic beads following the manufacturer's instructions (Miltenyi Biotech). Resulting cell populations were $95 \% \mathrm{CD}^{+}$-positive according to FACS analysis. Viability at culture harvest for analysis averaged $82 \%$. 


\section{ELISPOT assays}

Multi-screen HA plates (Millipore, MAHAS4510) were coated with $4-10 \mathrm{ug} / \mathrm{mL}$ of monoclonal capture $\mathrm{Ab}$ anti-human IFNy (1-D1K), IL-2 (IL-2-1), IL-10 (9D7) or TNFa (TNF3/4; all from MabTech), in PBS overnight at $4^{\circ} \mathrm{C}$. After blocking the plates with RPMI $/ 10 \% \mathrm{AB}(1 \mathrm{~h}$, $\left.4^{\circ} \mathrm{C}\right), \mathrm{CD}^{+}$or $\mathrm{CD} 4^{+} \mathrm{T}$ cells were plated at $10^{5}$ cells/well in duplicate or triplicate wells. Autologous DC $\left(0.5-1 \times 10^{5}\right.$ well) were pulsed with different peptides or transduced with HAdV-5, rinsed and plated. Control wells contained $\mathrm{T}$ cells (alone or with $0.2 \mathrm{ng} / \mathrm{ml} \mathrm{PMA}+0.2 \mu \mathrm{M}$ ionomycin), T cells with unloaded DC ("no peptide") or negative antigen (HIV gag or alpha fetoprotein peptides tested in a subset of ELISPOT assays) pulsed DC. Cells were removed and captured cytokine was detected by corresponding biotinylated $\mathrm{mAb}$ (MabTech) at $2 \mu \mathrm{g} / \mathrm{mL}$ in PBS/0.5\% BSA. After washing, Avidin Peroxidase Complex (Vectastain Elite Kit) was added for $60 \mathrm{~min}$. After rinsing, peroxidase staining was performed with 3-amino-9-ethylcarbazole (AEC, Sigma) and stopped by rinsing the plates under tap water. Spot numbers were automatically determined with ImmunoSpot imaging system from Cellular Technology, Ltd. Spots detected to "medium only" or T cells only were $<10$. Spots detected to PMA + ionomycin were $>1,000$ on average. To calculate the number of responding $\mathrm{T}$ cells, the mean number of spots detected with DC alone were subtracted from mean spot numbers induced by antigen-loaded DC. The range of DC only background subtracted varied by cytokine examined.

\section{Neutralizing antibody assay}

Anti-HAdV-5 neutralizing antibodies were detected using serial dilutions of serum (1:4 to 1:512) cultured with an indicator cell line, A549 (ATCC), followed by transduction with an HAdV-5-encoding enhanced Green Fluorescent Protein (HAdV-5 eGFP), as described [36]. The cells were tested for the MFI of eGFP by flow cytometry.

\section{Luminex assay}

Cell-free supernatants were collected from cultures and frozen at $-80^{\circ} \mathrm{C}$. They were subsequently thawed and simultaneously analyzed with the multiplex Luminex assay (Invitrogen) per manufacturer's protocol in a BioRad reader (UPCI Immunologic Monitoring Laboratory). The following analytes were tested: GM-CSF, IFN $\gamma$, IP-10, MCP-1, TNF $\alpha$, IL-1 $\beta$, IL-2, IL-4, IL-5, IL-6, IL-8, and IL-10, IL-15,IL-13, IL-17, IL-1R $\alpha$, MIP-1 $\beta$, IL-2r,IL-7 Exotaxin, MCP-1, MIG, MIP-1alpha and RANTES, in a kit pre-tested for any potential cross-reactivity by the manufacturer. Controls included the standard curve and multiplex QC standards (R\&D Systems).

\section{Additional files}

Additional file 1: Figure S1. Anti-HAdV-5 neutralizing antibodies. Serum from each $\mathrm{HD}$ and patient was tested for the level of neutralizing antibodies to HAdV-5. Assay controls of no HAdV-5 (negative), no serum (positive) and pooled human $A B$ serum are also shown. GFP expression in the A549 indicator cells is shown as MFI. The dilution of serum is on the $X$ axis and the extent of HAdV-5eGFP transduction blockade is plotted.

Additional file 2: Figure S2. NK cells activated and expanded in vitro. The CD56/CD16 gating strategy (of gated lymphocytes by forward and size scatter, not shown) is shown in $A$, and the number in each gate corresponds to the population analyzed in B and C. The cultured cells were assessed weekly, and the frequency of activated $\left(\mathrm{CD} 9^{+}\right) \mathrm{NK}$ cells from each gate is shown in $\mathrm{B}$, and the subset of those $\mathrm{CD} 69^{+}$cells expressing IFNy is shown in C. Error bars represent standard errors.

\section{Abbreviations}

PBMC: Peripheral blood mononuclear cells; AFP: Alpha fetoprotein; DC: Dendritic cells; Treg: Regulatory T cells; NK: Natural killer; HAdV-5: Human adenovirus serotype 5; MFI: Mean fluorescence intensity; HAdV-5 TMM2: Human adenovirus serotype 5/Tyrosinase, MART-1, MAGE-A6; HD: Healthy donors.

\section{Competing interests}

The authors declare that they have no competing interests.

\section{Authors' contributions}

HPN performed the experiments, analyzed the data and wrote the manuscript; $\mathrm{LV}$ assisted with aspects of the study and with writing the manuscript. LH Butterfield conceived of the study, supervised its conduct, reviewed the data and wrote the manuscript. All authors read and approved the final manuscript.

\section{Authors' information}

Hadas Prag Naveh is a Dermatologist currently at Department of Dermatology, Rabin Medical Center, Beilinson Hospital, Israel. Lazar Vujanovic is a Research Instructor in Medicine, and Lisa H. Butterfield is a Professor of Medicine, Surgery, and Immunology at the University of Pittsburgh Cancer Institute (UPCI) and the University of Pittsburgh.

\section{Acknowledgements}

SPORE P50 CA121973, CCSG P30 CA047904, UPCI Immunologic Monitoring Laboratory (Mary Jo Buffo, Seth Shaltes, Jennifer Muzzio, Sylvia Thomas-Shrader, Cathy Brown), Jian Shi, MD for HAdV-5 TMM2 preparation and testing, University of Pittsburgh Vector Core, University of Pittsburgh Peptide Synthesis Facility.

\section{Author details}

${ }^{1}$ Department of Medicine, University of Pittsburgh Cancer Institute, 5117 Centre Avenue, Suite 1.27, Pittsburgh, PA 15213, USA. ²Department of Surgery, University of Pittsburgh Cancer Institute, 5117 Centre Avenue, Suite 1.27, Pittsburgh, PA 15213, USA. ${ }^{3}$ Department of Immunology, University of Pittsburgh Cancer Institute, 5117 Centre Avenue, Suite 1.27, Pittsburgh, PA 15213, USA. ${ }^{4}$ University of Pittsburgh Cancer Institute, 5117 Centre Avenue, Suite 1.27, Pittsburgh, PA 15213, USA.

Received: 25 June 2013 Accepted: 13 November 2013 Published: 18 November 2013

\section{References}

1. Lasaro MO, Ertl HC: New insights on adenovirus as vaccine vectors. Molecular Ther 2009, 17:1333-1339.

2. Campos SK, Barry MA: Current advances and future challenges in Adenoviral vector biology and targeting. Curr Gene Ther 2007, 7:189-204

3. Butterfield LH, Vujanovic L: New approaches to the development of adenoviral dendritic cell vaccines in melanoma. Curr Opin Investig Drugs 2010, 11:1399-1408.

4. Butterfield LH, Jilani SM, Chakraborty NG, Bui LA, Ribas A, Dissette VB, Lau R, Gamradt SC, Glaspy JA, McBride WH, et al: Generation of melanoma-specific cytotoxic T lymphocytes by dendritic cells transduced with a MART-1 adenovirus. J Immunol 1998, 161:5607-5613. 
5. Perez-Diez A, Butterfield LH, Li L, Chakraborty NG, Economou JS, Mukherji B: Generation of CD8+ and CD4+ T-cell response to dendritic cells genetically engineered to express the MART-1/Melan-A gene. Cancer Res 1998, 58:5305-5309.

6. Butterfield LH, Comin-Anduix B, Vujanovic L, Lee Y, Dissette VB, Yang JQ, Vu HT, Seja E, Oseguera DK, Potter DM, et al: Adenovirus MART-1-engineered autologous dendritic cell vaccine for metastatic melanoma. J Immunother 2008, 31:294-309.

7. Liu Y, Daley S, Evdokimova VN, Zdobinski DD, Potter DM, Butterfield LH: Hierarchy of alpha fetoprotein (AFP)-specific T cell responses in subjects with AFP-positive hepatocellular cancer. J Immunol 2006, 177:712-721.

8. Evdokimova VN, Liu Y, Potter DM, Butterfield LH: AFP-specific CD4+ helper T-cell responses in healthy donors and HCC patients. J Immunother 2007, 30:425-437.

9. Butterfield LH, Ribas A, Potter DM, Economou JS: Spontaneous and vaccine induced AFP-specific T cell phenotypes in subjects with AFP-positive hepatocellular cancer. Cancer Immunol Immunother 2007, 56:1931-1943.

10. Schumacher L, Ribas A, Dissette VB, McBride WH, Mukherji B, Economou JS, Butterfield LH: Human dendritic cell maturation by adenovirus transduction enhances tumor antigen-specific T-cell responses. $J$ Immunother 2004, 27:191-200.

11. Vujanovic L, Whiteside TL, Potter DM, Chu J, Ferrone S, Butterfield LH: Regulation of antigen presentation machinery in human dendritic cells by recombinant adenovirus. Cancer Immunol Immunother 2009, 58:121-133.

12. Vujanovic L, Szymkowski DE, Alber S, Watkins SC, Vujanovic NL, Butterfield $\mathrm{LH}$ : Virally infected and matured human dendritic cells activate natural killer cells via cooperative activity of plasma membrane-bound TNF and IL-15. Blood 2010, 116:575-583.

13. Vujanovic L, Ballard W, Thorne SH, Vujanovic NL, Butterfield LH: Adenovirusengineered human dendritic cells induce natural killer cell chemotaxis via CXCL8/IL-8 and CXCL10/IP-10. Oncoimmunology 2012, 1:448-457.

14. Ribas A, Butterfield LH, Hu B, Dissette VB, Meng WS, Koh A, Andrews KJ, Lee M, Amar SN, Glaspy JA, et al: Immune deviation and Fas-mediated deletion limit antitumor activity after multiple dendritic cell vaccinations in mice. Cancer Res 2000, 60:2218-2224.

15. Buchbinder SP, Mehrotra DV, Duerr A, Fitzgerald DW, Mogg R, Li D, Gilbert PB, Lama JR, Marmor M, Del Rio C, et al: Efficacy assessment of a cell-mediated immunity HIV-1 vaccine (the Step Study): a double-blind, randomised, placebo-controlled, test-of-concept trial. Lancet 2008, 372:1881-1893.

16. Koup RA, Lamoreaux L, Zarkowsky D, Bailer RT, King CR, Gall JG, Brough DE, Graham BS, Roederer M: Replication-defective adenovirus vectors with multiple deletions do not induce measurable vector-specific T cells in human trials. J Virol 2009, 83:6318-6322.

17. Pine SO, Kublin JG, Hammer SM, Borgerding J, Huang Y, Casimiro DR, McElrath MJ: Pre-existing adenovirus immunity modifies a complex mixed Th1 and Th2 cytokine response to an Ad5/HIV-1 vaccine candidate in humans. PloS One 2011, 6:e18526.

18. Sukdolak C, Tischer S, Dieks D, Figueiredo C, Goudeva L, Heuft HG, Verboom M, Immenschuh S, Heim A, Borchers S, et al: CMV-, EBV- and ADV-specific T Cell immunity: screening and monitoring of potential third-party donors to improve post-transplantation outcome. Biol Blood Marrow Transplant 2013, 19:1480-1492.

19. Gerdemann U, Katari UL, Papadopoulou A, Keirnan JM, Craddock JA, Liu H, Martinez CA, Kennedy-Nasser A, Leung KS, Gottschalk SM, et al: Safety and clinical efficacy of rapidly-generated trivirus-directed T cells as treatment for adenovirus, EBV, and CMV infections after allogeneic hematopoietic stem cell transplant. Mol Ther 2013, 21(11): 2113-21.

20. Leen AM, Myers GD, Sili U, Huls MH, Weiss H, Leung KS, Carrum G, Krance RA, Chang CC, Molldrem JJ, et al: Monoculture-derived T lymphocytes specific for multiple viruses expand and produce clinically relevant effects in immunocompromised individuals. Nat Med 2006, 12:1160-1166.

21. Feuchtinger $T$, Richard $C$, Joachim S, Scheible MH, Schumm M, Hamprecht K, Martin D, Jahn G, Handgretinger R, Lang P: Clinical grade generation of hexon-specific T cells for adoptive T-cell transfer as a treatment of adenovirus infection after allogeneic stem cell transplantation. J Immunother 2008, 31:199-206.

22. Tsao H, Millman P, Linette GP, Hodi FS, Sober AJ, Goldberg MA, Haluska FG Hypopigmentation associated with an adenovirus-mediated gp100/ MART-1-transduced dendritic cell vaccine for metastatic melanoma. Archives of dermatology 2002, 138:799-802.
23. Haluska F, Linette G, Jonash S, Hodi S, Longerich S, Yang S, Webb I, Stowell C, Kaplan J, Roberts B, Goldberg M: Immunologic gene therapy of melanoma: phase I study of therapy with dendritic cells transduced with recombinant adenoviruses encoding melanoma antigens (Abstract 1777). Proc Am Soc Clin Oncol (Abstract) 2000, 19:453a.

24. Blalock LT, Landsberg J, Messmer M, Shi J, Pardee AD, Haskell R, Vujanovic L, Kirkwood JM, Butterfield LH: Human dendritic cells adenovirally-engineered to express three defined tumor antigens promote broad adaptive and innate immunity. Oncoimmunology 2012, 1:287-357.

25. Tang J, Murtadha M, Schnell M, Eisenlohr LC, Hooper J, Flomenberg P: Human T-cell responses to vaccinia virus envelope proteins. Journal of virology 2006, 80:10010-10020.

26. Olive M, Eisenlohr L, Flomenberg N, Hsu S, Flomenberg P: The adenovirus capsid protein hexon contains a highly conserved human CD4+ T-cell epitope. Human gene therapy 2002, 13:1167-1178.

27. Butterfield LH, Ribas A, Dissette VB, Amarnani SN, Vu HT, Oseguera D, Wang HJ, Elashoff RM, McBride WH, Mukherji B, et al: Determinant spreading associated with clinical response in dendritic cell-based immunotherapy for malignant melanoma. Cell Growth Differ 2003, 9:998-1008.

28. Ribas A, Glaspy JA, Lee Y, Dissette VB, Seja E, Vu HT, Tchekmedyian NS, Oseguera D, Comin-Anduix B, Wargo JA, et al: Role of dendritic cell phenotype, determinant spreading, and negative costimulatory blockade in dendritic cell-based melanoma immunotherapy. J Immunother 2004, 27:354-367.

29. Huarte E, Larrea E, Hernandez-Alcoceba R, Alfaro C, Murillo O, Arina A, Tirapu I, Azpilicueta A, Hervas-Stubbs S, Bortolanza S, et al: Recombinant adenoviral vectors turn on the type I interferon system without inhibition of transgene expression and viral replication. Mol Ther 2006, 14:129-138.

30. Whiteside TL, Zhao Y, Tsukishiro T, Elder EM, Gooding W, Baar J: Enzyme-linked immunospot, cytokine flow cytometry, and tetramers in the detection of T-cell responses to a dendritic cell-based multipeptide vaccine in patients with melanoma. Clin Cancer Res 2003, 9:641-649.

31. Poli A, Michel T, Theresine M, Andres E, Hentges F, Zimmer J: CD56bright natural killer (NK) cells: an important NK cell subset. Immunology 2009, 126:458-465.

32. Steinman RM, Hawiger D, Nussenzweig MC: Tolerogenic dendritic cells. Annu Rev Immunol 2003, 21:685-711.

33. Fischer HG, Frosch S, Reske K, Reske-Kunz AB: Granulocyte-macrophage colony-stimulating factor activates macrophages derived from bone marrow cultures to synthesis of MHC class II molecules and to augmented antigen presentation function. J Immunol 1988, 141:3882-3888.

34. Everly JJ, Lonial S: Immunomodulatory effects of human recombinant granulocyte-macrophage colony-stimulating factor (rhuGM-CSF): evidence of antitumour activity. Expert Opin Biol Ther 2005, 5:293-311.

35. van Leeuwen EM, Sprent J, Surh CD: Generation and maintenance of memory CD4(+) T cells. Curr Opin Immunol 2009, 21:167-172.

36. Nwanegbo E, Vardas E, Gao W, Whittle H, Sun H, Rowe D, Robbins PD, Gambotto A: Prevalence of neutralizing antibodies to adenoviral serotypes 5 and 35 in the adult populations of The Gambia, South Africa, and the United States. Clin Diagn Lab Immunol 2004, 11:351-357.

37. Johnson MJ, Petrovas C, Yamamoto T, Lindsay RW, Lore K, Gall JG, Gostick E, Lefebvre F, Cameron MJ, Price DA, et al: Type I IFN induced by adenovirus serotypes 28 and 35 has multiple effects on T cell immunogenicity. $\mathrm{J}$ Immunol 2012, 188:6109-6118.

38. Tuting T, Wilson CC, Martin DM, Kasamon YL, Rowles J, Ma DI, Slingluff CL Jr, Wagner SN, van der Bruggen P, Baar J, et al: Autologous human monocyte-derived dendritic cells genetically modified to express melanoma antigens elicit primary cytotoxic $\mathrm{T}$ cell responses in vitro: enhancement by cotransfection of genes encoding the Th1-biasing cytokines IL-12 and IFN-alpha. J Immunol 1998, 160:1139-1147.

39. Fridman WH, Pages F, Sautes-Fridman C, Galon J: The immune contexture in human tumours: impact on clinical outcome. Nat Rev Cancer 2012, 12:298-306.

40. Braumuller $\mathrm{H}$, Wieder $\mathrm{T}$, Brenner E, Assmann S, Hahn M, Alkhaled M, Schilbach K, Essmann F, Kneilling M, Griessinger C, et al: T-helper-1-cell cytokines drive cancer into senescence. Nature 2013, 494:361-365.

doi:10.1186/2051-1426-1-19

Cite this article as: Naveh et al:: Cellular immunity induced by a recombinant adenovirus- human dendritic cell vaccine for melanoma. Journal for ImmunoTherapy of Cancer 2013 1:19. 